\title{
Hydrogen/Deuterium Exchange on Yeast ATPase Supramolecular Protein Complex Analyzed at High Sensitivity by MALDI Mass Spectrometry
}

\author{
Alexis Nazabal, Michel Laguerre, and Jean-Marie Schmitter \\ Institut Européen de Chimie et Biologie, Pessac, France \\ Jacques Vaillier, Stéphane Chaignepain, and Jean Velours \\ Institut de Biologie et Génétique Cellulaire, Bordeaux, France
}

To evaluate the ability of hydrogen/deuterium exchange of amide protons followed by mass spectrometry (HXMS) to yield topological information about supramolecular protein complexes, this approach has been tested with the $370 \mathrm{kDa}$ hetero-oligomeric complex of yeast F1-ATPase. The study was focused on the $\varepsilon$ subunit $(6612 \mathrm{Da}$ ) of the complex. Deuterium back exchange due to the chromatographic isolation step of this subunit was strongly reduced by means of fast micro-chromatography, and MALDI-MS was used to analyze either the intact subunit or peptide mixtures resulting from its proteolytic cleavage. A deuterium labeling kinetic study was conducted with $\varepsilon$ subunit being a part of the F1 native complex. The effect of a secondary structure was also investigated by means of HXMS on the isolated $\varepsilon$ subunit. Finally, to determine which regions of $\varepsilon$ subunit are accessible to solvent in F1-ATPase during exchange, the complex was submitted to hydrogen/deuterium exchange, the $\varepsilon$ subunit was purified by micro-chromatography, digested by pepsin, and resulting peptide fragments were analyzed by MALDI-MS. The combination of hydrogen/deuterium exchange, fast microchromatography and MALDI-MS was shown to be a fast and efficient way to obtain detailed topological information for the $\varepsilon$ subunit when it is engaged in the ATPase complex. (J Am Soc Mass Spectrom 2003, 14, 471-481) @ 2003 American Society for Mass Spectrometry

I n native proteins, hydrogen/deuterium (H/D) exchange of amide protons not only depends on solvent accessibility and spatial arrangement of amino acid residues into structure elements such as $\alpha$-helices and $\beta$-sheets, but also on the supramolecular organization of proteins. Thus, the analysis of H/D exchange may yield a fingerprint of the 3-D structure of a protein as well as a fingerprint of its direct environment within a supramolecular complex [1, 2].

Hydrogen/deuterium exchange monitored by mass spectrometry (HXMS) allows the detection of isotopic exchange at backbone amide positions of proteins $(\mathrm{H}$ atoms covalently bound to carbon do not exchange, whereas $\mathrm{H}$ atoms on side chains exchange too fast to be evidenced by this methodology), and has been used to investigate various aspects of protein structure [3-5].

A major difficulty associated to HXMS methodology is deuterium back exchange. To limit this phenomenon

Published online April 3, 2003

Address reprint requests to A. Nazabal, Institut Européen de Chimie et Biologie, CNRS FRE 2247, 16, Avenue Pey Berland, Pessac Cedex 33607, France. E-mail: a.nazabal@iecb-polytechnique.u-bordeauxfr

after an exchange usually performed under native conditions, the reaction must be quenched $\left(\mathrm{pH} 2,0^{\circ} \mathrm{C}\right)$ and the samples analysis has to be performed quickly under these last conditions [6]. To gain access to the distribution of exchanged deuterons over the protein sequence, peptides are generated by proteolytic cleavage with pepsin. This enzyme works properly at low $\mathrm{pH}$, but its lack of specificity induces constraints for mass spectrometric peptide assignments.

Although many HXMS studies have been conducted with electrospray ionization (ESI), matrix-assisted laser desorption/ionization mass spectrometry (MALDI-MS) turns out to be a convenient tool for this purpose, taking advantage of its high speed of analysis, excellent sensitivity, and accuracy [7,8]. Furthermore, the analysis of peptide maps by MALDI-MS after pepsin digestion neither requires combined liquid chromatographymass spectrometry, nor tedious back exchange corrections $[9,10]$.

One step further, solvent accessibility of subunits belonging to a hetero-oligomeric supramolecular protein complex may also be investigated by means of 
HXMS. However, such experiments require an additional step, because the subunits of interest must be isolated by means of liquid chromatography, and again back exchange must be kept as low as possible during this separation step.

To evaluate if HXMS may lead to a precise characterization of the solvent accessibility of a given subunit within a hetero-oligomeric complex, we report here a topological study of yeast F1-ATPase, a $370 \mathrm{kDa}$ complex. The F1 domain is the catalytic sector of ATP synthase, a mitochondrial enzyme responsible for ATP synthesis. F1 domain comprises five different subunits: $\alpha(55.3 \mathrm{kDa}), \beta(52.5 \mathrm{kDa}), \gamma(30.6 \mathrm{kDa}), \delta(14.5 \mathrm{kDa})$, and $\varepsilon(6.6 \mathrm{kDa})$, in 3:3:1:1:1 stoichiometry. We have chosen to focus our study on the $\varepsilon$ subunit (61 amino acids long, $6612 \mathrm{Da}$ average mass, for the mature protein having undergone methionine excision) [11].

A fast micro-chromatographic method has been designed in order to disrupt the F1-ATPase complex and to isolate $\varepsilon$ from the other subunits in less than one min. HXMS studies were performed first with the $\varepsilon$ subunit isolated in this way. The extent of H/D exchange could be determined by MALDI-MS measurement of the average mass of the whole subunit. The effect of the fast micro-chromatographic purification step on deuterium back exchange was evaluated. To study the effect of the secondary structure of this subunit on H/D exchange, deuterium labeling was measured using various solvent conditions.

A kinetic HXMS study was then realized on $\varepsilon$ subunit while embedded in F1-ATPase. To determine which regions of $\varepsilon$ subunit are accessible to solvent in F1-ATPase, H/D exchange of the whole F1-ATPase complex was followed by a micro-chromatographic isolation of the $\varepsilon$ subunit, which was then submitted to a proteolytic cleavage using pepsin, prior to MALDI-MS analysis of the resulting peptides. Owing to the poor specificity of pepsin, the critical point of the methodology lies in the requirement for a complete sequence coverage of the subunit of interest, with all peptides reliably assigned. Thus, peptide ions selected for our study were either unambiguously attributed to a given stretch from their mass values, or were assigned after fragment ion analysis [MALDI post source decay (PSD) fragmentation or ESI/MS/MS analysis by means of an ion trap].

Results were evaluated with the help of the 3-D model structure of the bovine enzyme. The possible extension of this MALDI-HXMS methodology to larger subunits belonging to supramolecular complexes is discussed.

\section{Experimental}

Deuterium oxyde (99.9\%) was purchased from Eurisotop (Gif-sur-Yvette, France). Sequence grade trifluoroacetic acid (TFA) and immobilized pepsin were obtained from Pierce (Rockford, IL). Acetonitrile (HPLC grade) was purchased from Mallinckrodt Baker, (De- venter, The Netherlands). ZipTip $C_{18}$ pipette tips were purchased from Millipore (Bedford, MA). $\mathrm{pH}$ Indicator strips were obtained from Microessential Laboratory, (New York, NY). Polypropylene micro-centrifuge tubes were from Treff (Degersheim, Switzerland). Folin and Ciocalteu's phenol reagent was obtained from Sigma (Saint Louis, MO). Water was purified over a MilliQ apparatus (Millipore), and is hereafter referred as MQ grade.

\section{Purification of Saccharomyces cerevisiae F1-ATPase}

Mitoplasts were prepared according to Daum et al. [12] with slight modifications. A suspension of mitochondria $(20 \mathrm{mg}$ of protein $/ \mathrm{ml}$ in $0.6 \mathrm{M}$ mannitol, $2 \mathrm{mM}$ EGTA, $10 \mathrm{mM}$ p-aminobenzamidine (PAB), $10 \mathrm{mM}$ $\varepsilon$-amino-n-caproic acid, $0.5 \mathrm{mM}$ phenylmethylsulfonyl fluoride, $10 \mathrm{mM}$ Tris-maleate, $\mathrm{pH}$ 6.8) was diluted with 5 volumes of the same buffer without mannitol. The suspension of mitochondria was stirred very gently on a magnetic stirrer at $4^{\circ} \mathrm{C}$ for $20 \mathrm{~min}$. The suspension was sedimented at $4{ }^{\circ} \mathrm{C}(48,000 \mathrm{~g}$ for $20 \mathrm{~min})$. The pellet was kept frozen overnight at $-80^{\circ} \mathrm{C}$. Mitochondrial membranes were thawed at $4{ }^{\circ} \mathrm{C}$ and resuspended in the same buffer as above to a protein concentration of 2 $\mathrm{mg} / \mathrm{ml}$. After $5 \mathrm{~min}$, the suspension was exposed twice to ultrasonic irradiation (Annemasse sonicator, $30 \mathrm{~s}$ at $120 \mathrm{~V})$. Total mitochondrial membranes were sedimented at $4{ }^{\circ} \mathrm{C}(45 \mathrm{~min}$ at $100,000 \mathrm{~g})$. Extraction by chloroform was used to remove F1-ATPase from mitochondrial membranes [13].

Purification of yeast F1-ATPase was according to Penin et al. [14] and Falson et al. [15] with some modifications. The mitochondrial membranes were homogenized in $0.3 \mathrm{M}$ mannitol, $1 \mathrm{mM}$ EDTA, $10 \mathrm{mM}$ PAB, $10 \mathrm{mM} \varepsilon$-amino-n-caproic acid, $0.5 \mathrm{mM}$ phenylmethylsulfonyl fluoride, $2 \mathrm{mM}$ ATP, $10 \mathrm{mM}$ Tris-maleate, $\mathrm{pH} 7.0$ to a final concentration of $10 \mathrm{mg} / \mathrm{ml}$. The suspension was firmly shaken in the presence of half a volume of chloroform for $20 \mathrm{~s}$ at room temperature. The aqueous phase was recovered after centrifugation at $1.100 \mathrm{~g}$ for $10 \mathrm{~min}$ at $20^{\circ} \mathrm{C}$, and was centrifuged again at $100,000 \mathrm{~g}$ for $30 \mathrm{~min}$ at $20^{\circ} \mathrm{C}$. EDTA was added to the supernatant to a final concentration of $4 \mathrm{mM}$. The solution was maintained at $\mathrm{pH} 7$ by $\mathrm{NaOH}$ addition, brought to $70 \%$ saturation with solid ammonium sulfate and stored at $4{ }^{\circ} \mathrm{C}$.

The ammonium sulfate suspension was centrifuged at $100,000 \mathrm{~g}$ for $45 \mathrm{~min}$ at $4{ }^{\circ} \mathrm{C}$. The pellet was solubilized in a minimal volume of $50 \%$ (wt/vol) glycerol, 5 mM EDTA, 10 mM PAB, 100 mM Tris-HCl, pH 7.5, and centrifuged twice in an Eppendorf centrifuge tube to remove potential aggregates. The supernatant was applied to a small Sephadex (Amersham Biosciences, Freiburg, Germany) G-50 (fine grade) centrifuge column that had been equilibrated with $50 \%$ (wt/vol) glycerol, $5 \mathrm{mM}$ EDTA, $100 \mathrm{mM}$ Tris- $\mathrm{HCl}, \mathrm{pH}$ 7.5, 
according to Penefsky [16]. The eluate was submitted to high performance gel permeation chromatography over a TSK Spherogel SW 4000 column $(0.7 \mathrm{~cm}$ i.d., $30 \mathrm{~cm}$ long, Altex, Beckman Coulter Inc., Fullerton, CA), equilibrated and eluted at room temperature with the above buffer, without $\mathrm{PAB}$, at a flow rate of $0.3 \mathrm{ml} / \mathrm{min}$. Detection was monitored at $280 \mathrm{~nm}$. The fraction of eluate containing the enzyme was concentrated by means of ultracentrifugation membranes and stored as ammonium sulfate precipitate.

\section{F1-ATPase Assay}

Purified and concentrated F1-ATPase (8-10 $\mu \mathrm{g})$ was incubated at $30{ }^{\circ} \mathrm{C}$ for $2 \mathrm{~min}$ in $900 \mu \mathrm{l}$ of a reaction mixture containing $50 \mathrm{mM}$ Tris $/ \mathrm{SO}_{4} \mathrm{pH} 8.5,4 \mathrm{mM}$ $\mathrm{MgSO}_{4}$ in $\mathrm{D}_{2} \mathrm{O}$. The reaction was started with $50 \mu \mathrm{l}$ of $0.1 \mathrm{M} \mathrm{ATP} \mathrm{pH} \mathrm{7}$, allowed to proceed for $2 \mathrm{~min}$ and stopped by addition of $100 \mu \mathrm{l}$ of trichloroacetic acid. The mixture was centrifuged for $7 \mathrm{~min}$ at 10,000 g. An $0.5 \mathrm{ml}$ aliquot of the deproteinized supernatant was used for the colorimetric quantification of released inorganic phosphate. This aliquot was mixed with 2.5 $\mathrm{ml}$ of $0.75 \mathrm{~N}$ sulfonic acid, $1 \mathrm{mM}$ ferric sulfate and 0.1 $\mathrm{mM}$ heptamolybdate, and incubated at room temperature for $15 \mathrm{~min}$. Inorganic phosphate concentration was determined spectrophotometrically at $610 \mathrm{~nm}$.

\section{Fast Isolation of $\varepsilon$ Subunit from F1-ATPase}

The fast isolation of $\varepsilon$ subunit from F1-ATPase was obtained by micro-chromatography over a $\mathrm{C}_{18}$ ZipTip (15 $\mu \mathrm{m}$ particles, $20 \mathrm{~nm}$ pore size, $0.6 \mu \mathrm{l}$ stationary phase, Millipore). Before use, the $\mathrm{C}_{18}$ support was wetted with $70 \%$ acetonitrile in MQ water, $0.1 \%$ TFA (by aspiring and dispensing 5 times to waste) and the ZipTip was equilibrated in $\mathrm{H}_{2} \mathrm{O} \mathrm{MQ}, 0.1 \%$ TFA. $4 \mu \mathrm{l}$ of the F1-ATPase solution $(7 \mathrm{mg} / \mathrm{ml})$ were loaded. After binding, the support was washed $\left(\mathrm{H}_{2} \mathrm{O} \mathrm{MQ}, 0.1 \%\right.$ TFA, aspirated and dispensed to waste three times). The $\varepsilon$ subunit was eluted with $2 \mu \mathrm{l}$ of $20 \%$ acetonitrile in $\mathrm{H}_{2} \mathrm{O}$ MQ, $0.1 \%$ TFA. In this way, the isolation of $\varepsilon$ subunit from F1-ATPase was achieved in less than $1 \mathrm{~min}$.

\section{Back Exchange in Relation to Chromatographic Isolation of $\varepsilon$ Subunit}

An aliquot of $\varepsilon$ subunit, previously isolated by microchromatography, was dried completely, redissolved in labeling solvent $\left(\mathrm{D}_{2} \mathrm{O}\right.$, acetonitrile $\left.4: 1\right)$ and incubated 60 min at room temperature to achieve complete $H / D$ exchange of backbone amide protons. Isotopic exchange was quenched by mixing the sample with matrix ( $\alpha$ cyano-4-hydroxycinnamic acid in a solution containing 1:1:1 acetonitrile, ethanol, and 0.1\% TFA, pH 2, kept at $0{ }^{\circ} \mathrm{C}$ ). The incorporation of deuterium was directly determined by MALDI-MS analysis. To evaluate the effect of the chromatographic separation step on back exchange, the $\varepsilon$ subunit was submitted to fast microchromatography after $60 \mathrm{~min}$ incubation time. The collected fraction containing $\varepsilon$ was mixed with the matrix solution and the incorporation of deuterium was determined by MALDI-MS analysis and compared to the previous one.

\section{HXMS on Isolated $\varepsilon$ Subunit-Effect of Acetonitrile}

The $\varepsilon$ subunit solution isolated from F1-ATPase $(2 \mu \mathrm{l})$ was dried in its vial using a gentle nitrogen flow. Solutions used for H/D exchange had the following acetonitrile: $\mathrm{D}_{2} \mathrm{O}$ compositions: 20:80, 30:70, 40:60, and 70:30. The deuteration solution was added to the vial and sonicated for a few seconds. After the desired incubation time at room temperature, $1 \mu$ l of the sample was mixed with $1 \mu l$ of the matrix solution. The matrix used in these experiments was $10 \mathrm{mg} / \mathrm{ml} \alpha$-cyano-4hydroxycinnamic acid, in a solution containing 1:1:1 acetonitrile, ethanol, and $0.1 \%$ TFA in $\mathrm{H}_{2} \mathrm{O}$. The matrix solution was kept over ice and mixed by vortexing before use. Immediately after mixing, $0.7 \mu \mathrm{l}$ of the sample was spotted onto the target and dried by a nitrogen flow. After drying, the target was transferred as quickly as possible (about $1 \mathrm{~min}$ after quenching) to the mass spectrometer sample inlet.

\section{HXMS on F1 ATPase Complex}

A $10 \mu$ aliquot of the ammonium sulfate suspension of F1-ATPase $(7 \mathrm{mg} / \mathrm{ml})$ was centrifuged at 10,000 $g$ for 10 min and the pellet was dissolved in $20 \mu \mathrm{D}_{2} \mathrm{O}$. After the desired incubation time at $25^{\circ} \mathrm{C}, 4 \mu \mathrm{l}$ of $\mathrm{F} 1$ solution in $\mathrm{D}_{2} \mathrm{O}$ were submitted to micro-chromatography by means of a ZipTip. The equilibrating solution, the washing solution, and the eluent solution were kept over ice before use. After fast isolation, $\varepsilon$ subunit samples were prepared for mass analysis (see below).

\section{Proteolytic Cleavage by Pepsin}

The $\varepsilon$ subunit (20 pmol in $5 \mu$ l of $20 \%$ acetonitrile, $0.01 \%$ TFA, $0{ }^{\circ} \mathrm{C}$ ) was digested with $45 \mathrm{u}$ of immobilized pepsin (Pierce) in a final volume of $20 \mu \mathrm{l}$ of $0.1 \%$ TFA. After incubation $\left(10 \mathrm{~min}, 0^{\circ} \mathrm{C}\right)$, the digest was centrifuged $(10,000 \mathrm{~g}, 1 \mathrm{~min})$ to separate the beads from the peptide fragments. $1 \mu \mathrm{l}$ of the supernatant $(2 \mathrm{pmol})$ was mixed with the matrix and spotted on the MALDI target under nitrogen flow.

\section{Structure Prediction and Models}

Calculations were performed on a SGI Octane workstation running InsightII and Discover version 2000 (Accelrys Inc., San Diego, CA). Sequence homology building was performed within the Homology module. The sequence used for the yeast $\varepsilon$ subunit was referenced as 
SwissProt P21306 (http://www.expasy.ch). The 3-D structure used as reference for homology building was the bovine F1-ATPase structure of Gibbons et al. [17] (2.4 A resolution, PDB code: 1E79) in which the 47 first amino-acids of the $\varepsilon$ subunit have been solved (full length of 50 aminoacids, SwissProt accession number P05632).

Secondary structure predictions were obtained by means of the following softwares: JPRED (http://jura.ebi.ac.uk:8888/), PHD (http://cubic.bioc.columbia.edu/pp), PSIpred (http://insulin.brunel.ac.uk/ psiform.html), SSpro (http://promoter.ics.uci.edu/ BRNN-PRED), SAM-T99 (http://www.cse.ucsc.edu/ research/compbio/HMM-apps/T99-query.html), and PROF (http://www.aber.ac.uk/I P5phiwww/prof/ index.html).

Lipophilicity potential maps have been calculated and drawn around the model of the yeast $\varepsilon$ subunit by means of in-house software [18].

\section{Mass Spectrometry}

MALDI mass spectra were acquired on a Bruker Reflex III mass spectrometer equipped with a nitrogen laser (337 nm emission wavelength). Spectra were obtained by accumulating an average of 100 shots in the positive ion mode, while the laser spot was manually scanned over a surface area of about $0.2 \mathrm{~mm}^{2}$. Deflection of the low mass ions was used to enhance the target protein signal. Mass spectra of the whole $\varepsilon$ subunit were obtained in the linear mode $(20 \mathrm{kV}$ accelerating voltage). An external calibration was performed for each measurement with the singly and doubly charged ions of the unlabeled $\varepsilon$ subunit. Mass values given in the text correspond to $\mathrm{m} / \mathrm{z}$ of the centroid of the envelope of the mass peaks for $[\mathrm{M}+\mathrm{H}]^{+}$pseudo-molecular ions. Mass spectra of $\varepsilon$ subunit digests were acquired in the reflectron mode, using external calibration with a mixture of eight peptides covering a 900 to 3500 mass range. In this case, measured masses were monoisotopic $[\mathrm{M}+\mathrm{H}]^{+}$ions.

For post source decay analysis, the reflectron voltage was stepped down from $30 \mathrm{kV}$ in 10-12 steps in order to records all fragment ions, including immonium ions.

In order to control the assignment of peptides, MS/MS analysis of some peptide sequences was performed by means of an ion trap mass spectrometer operated in the electrospray mode (LCQ DecaXP, Thermo Finnigan, San Jose, CA) and interfaced to a Dionex-LC Packings (San Francisco, CA) chromatographic system $\left(\mathrm{C}_{18}\right.$ column, $75 \mu \mathrm{m}$ i.d., $150 \mathrm{~mm}$ long $)$.

\section{Results and Discussion}

\section{MALDI-HXMS Conditions}

MALDI-HXMS combines the advantages of high speed of analysis and excellent sensitivity and accuracy of mass measurements with the capability to analyze peptide maps in a single spectral acquisition. Back exchange may be neglected for MALDI-HXMS experiments, provided that the sample preparation allows a quick transition between the moment of exchange quenching and the transfer into the ion source of the spectrometer [9]. We have chosen to prepare the matrix in a 1:1:1 acetonitrile, ethanol, and $0.1 \%$ aqueous TFA mixture kept on ice, to apply a small sample volume $(0.7 \mu \mathrm{l})$ on the target, and to dry it under a nitrogen flow. In this way, the target was ready to load in the mass spectrometer vacuum lock in less than one min. Since we found that water condensation on a cold target did affect the sample crystallization process, and that much time was spent to eliminate this condensate prior to mass analysis without benefit in terms of back exchange minimization, we did not follow the recommendation of Mandell et al. [9] who kept the target on ice. A deuterated matrix is not required, since the H/D exchange occurring on the MALDI target within the ion source is slow (it takes several hours to observe 50\% back exchange on a fully deuterated peptide).

\section{Control of Back Exchange During Chromatographic Separation of $\varepsilon$ Subunit}

In order to conduct HXMS experiments on the F1ATPase complex, with focus on the $\varepsilon$ subunit embedded in this assembly, controls were performed to evaluate the impact of a chromatographic separation process on back exchange. For this, the maximal exchange level was measured first with an $\varepsilon$ subunit sample previously isolated by liquid chromatography: in this case, labeling reached $95 \%$ of exchangeable amide protons (57 out of 60 ). In a separate experiment, the isolated $\varepsilon$ subunit was incubated for $60 \mathrm{~min}$ in deuterium oxide, then submitted again to liquid chromatography. In this latter case, the level of back exchange resulting from the use of a reversed phase chromatographic system was found to be very high. Two different columns were tested for this purpose, using a water/acetonitrile gradient in $0.1 \%$ TFA with a sharp slope. The first one was a $\mathrm{C}_{18}$ grafted silica column (Vydac 238, Hesperia, CA, with monomeric bonding, $5 \mu \mathrm{m}$ particles, $30 \mathrm{~nm}$ pores, $4.6 \mathrm{~mm}$ i.d., and $50 \mathrm{~mm}$ length). The $\varepsilon$ subunit eluted after $10 \mathrm{~min}$ and the corresponding fraction was immediately analyzed by MALDI-MS, or kept frozen in liquid nitrogen prior to this analysis. The maximal H/D exchange measured in this case by MALDI-MS was $44 \%$. In a second attempt, a Poros R2 (Perseptive Biosystems, Framingham, MA) column was used (10 $\mu \mathrm{m}$ particles, $4.6 \mathrm{~mm} \times$ $50 \mathrm{~mm}$ ). This support tolerated higher flow rates and the $\varepsilon$ subunit eluted after $6 \mathrm{~min}$, but the exchange level was only slightly better (52\%). We then developed a micro-chromatographic gradient separation using a ZipTip $\left(\mathrm{C}_{18}\right.$ support, $0.6 \mu \mathrm{l}$ stationary phase in the tip, cf experimental part). In this case, the $\varepsilon$ subunit was isolated with a step gradient elution at $20 \%$ acetonitrile, 


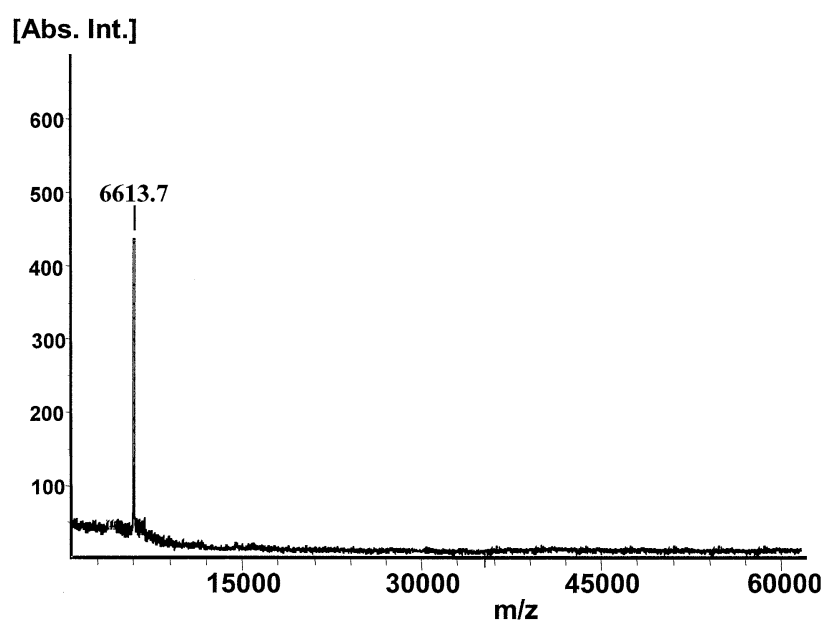

Figure 1. MALDI mass spectrum of $\varepsilon$ subunit isolated by reversed phase fast micro-chromatography. Out of the F1-ATPase complex (10 pmol in $0.04 \mathrm{M} \mathrm{MgSO}_{4}, 50 \mathrm{mM}$ Tris-SO ${ }_{4}$ in a $5 \mu \mathrm{l}$ volume), only the $\varepsilon$ subunit elutes from the C18 ZipTip at $20 \%$ acetonitrile concentration. One half of the elution fraction $(1 \mu \mathrm{l})$ was mixed with the matrix, spotted on the target and analyzed in the linear mode. The observed mass at $\mathrm{m} / \mathrm{z}$ 6613.7 Da corresponds to the $[\mathrm{M}+\mathrm{H}]^{+}$ion. The calculated average mass [M] for $\varepsilon$ subunit is $6612.6 \mathrm{Da}$.

allowing to separate it in less than one min from other F1 subunits, as shown by MALDI-MS analysis (Figure 1). In this way, the maximal exchange level was found to be $(92 \pm 3 \%)$ when the $\varepsilon$ subunit was incubated for various times (from $60 \mathrm{~min}$ to several h) before chromatographic separation.

This control was conducted with the isolated $\varepsilon$ subunit, but could not be performed on the whole F1-ATPase complex because it is not possible to determine in a single experiment if a measured decrease of the exchange level is due to back exchange, or if this decrease is due to interactions between subunits within the F1-ATPase complex.

\section{Incorporation of Deuterium in $\varepsilon$ Subunit as a Function of Incubation Time}

H/D exchange was allowed to occur on F1-ATPase with variable times between 2 and $2000 \mathrm{~min}$, followed by an isolation of the $\varepsilon$ subunit by means of fast micro-chromatography. Starting from a $\varepsilon$ subunit embedded in F1-ATPase, the deuterium content was found to increase rapidly during the first $20 \mathrm{~min}$ (Figure 2). After this incubation time, only a very slow variation of deuterium content was observed. This result indicates that a large part of the exchangeable hydrogen atoms of $\varepsilon$ subunit is still protected after 20 min of incubation in a deuterated solvent.

The exchange level can be quantified by means of the following equation [19]:

$$
\left(1 / \mathrm{H}_{\mathrm{t}}\right) \cdot \mathrm{H}=\mathrm{e}^{\left(-\mathrm{k}_{e x} \mathrm{t}\right)}
$$

with: (a)

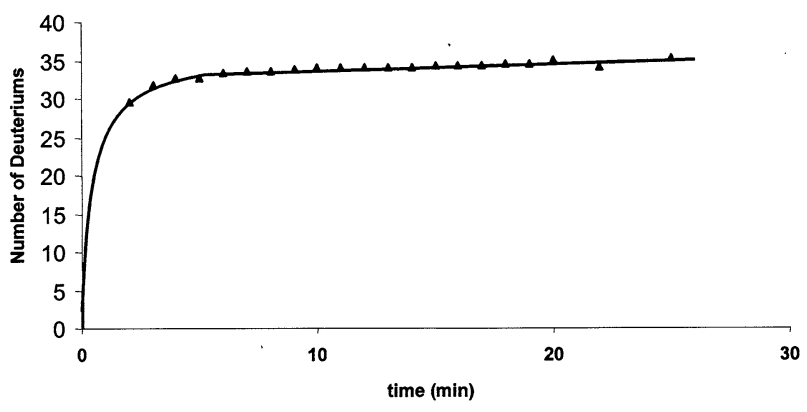

(b)

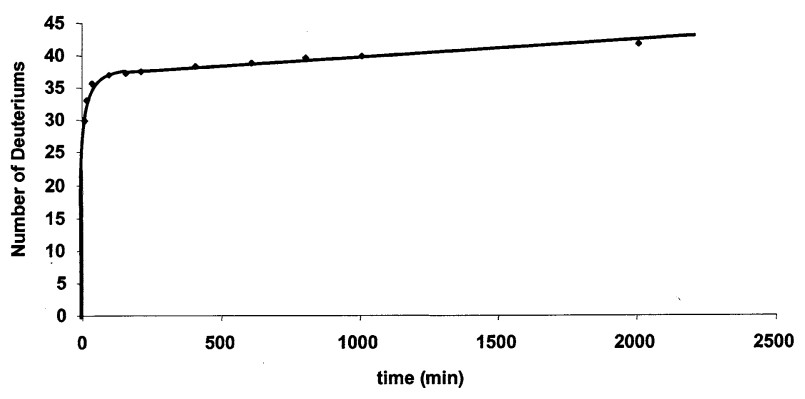

(c)

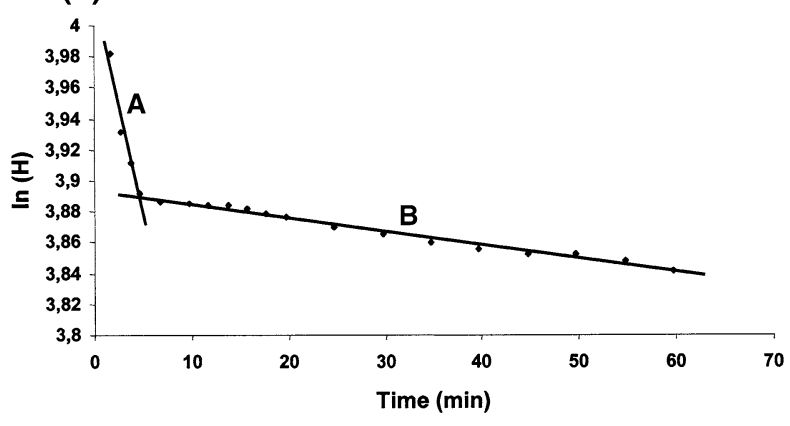

Figure 2. (a) Incorporation of deuterium in $\varepsilon$ subunit as a function of incubation time between 0 and $25 \mathrm{~min}$. After incubation in $\mathrm{D}_{2} \mathrm{O}, \varepsilon$ subunit was isolated from F1-ATPase and analyzed by MALDI-MS. Because no data are available for $\mathrm{t}<2 \mathrm{~min}$., the dotted line between 0 and $2 \mathrm{~min}$ is an extrapolation of the graph. (b) Same as above, for the 0 to 2000 min time interval. (c) Kinetics of H/D exchange of $\varepsilon$ subunit; the natural logarithm of the amount of protected hydrogens in $\varepsilon$ subunit $\ln (\mathrm{H})$ is plotted versus time (t). For the $\varepsilon$ subunit, intermediate exchange data are represented by the lines $(A) \operatorname{In}[\mathrm{H}]=-2.9 \times 10^{-2} \mathrm{t}+4.0\left(\mathrm{r}^{2}=0.93\right)$ and $(\mathbf{B}) \ln (\mathrm{H})=-9.0 \times 10^{-4} \mathrm{t}+3.9\left(\mathrm{r}^{2}=0.98\right)$.

- $\mathrm{H}_{\mathrm{t}}$ : Total amount of exchangeable hydrogen atoms

- $\mathrm{H}$ : Number of hydrogens present after time $t$.

- $\mathrm{k}_{e x}: \mathrm{H} / \mathrm{D}$ exchange rate constant

- $\mathrm{t}$ : Incubation time in $\mathrm{D}_{2} \mathrm{O}$.

Half-lives of exchangeable hydrogen atoms can be calculated from the plot $\ln [\mathrm{H}]$ versus $\mathrm{t}$ [20], which may also reveal populations of hydrogen atoms having different exchange rate constants. In our HXMS experiments, fast exchanging $\mathrm{H}$ atoms (between 0 and $2 \mathrm{~min}$ ) were already exchanged at the time of the first mea- 
surements. These hydrogen atoms are most accessible to the solvent and are not protected by hydrogen bonding involved in secondary structuration or supramolecular interactions of the $\varepsilon$ subunit within the complex. This accounts for about 29 amide protons for the $\varepsilon$ subunit in F1-ATPase (Figure 2a). The category corresponding to intermediate exchange (between 2 and $1000 \mathrm{~min}$ ) comprises $10 \mathrm{H}$ atoms (Figure 2b, $39 \mathrm{D}$ atoms exchanged at $1000 \mathrm{~min}$ ). As shown on Figure 2c, two populations may be distinguished in our case for this intermediate exchange. The first subpopulation is composed of $3 \mathrm{H}$ atoms with a half-life of $24 \mathrm{~min}$. The second subpopulation is composed of $7 \mathrm{H}$ atoms with a half-life of $69 \mathrm{~min}$. This leaves two slowly exchangeable $\mathrm{H}$ atoms (41 D atoms exchanged at $2000 \mathrm{~min}$, Figure 2b), that are highly protected.

This study of H/D exchange highlights the importance of the protecting effect due to interactions between the subunits. When the $\varepsilon$ subunit is embedded in the F1 complex, secondary structure and interactions with the neighboring $\gamma$ and $\delta$ subunits result in a protection of various exchangeable positions.

\section{Effect of $\varepsilon$ Subunit Secondary Structure on Deuterium Labeling}

In this experiment, $\varepsilon$ subunit was first isolated from F1-ATPase using fast micro-chromatography. After an incubation time of $10 \mathrm{~min}$ in deuterated solvent, samples were analyzed by MALDI-MS. We have shown that the maximal exchange level observed for an isolated $\varepsilon$ subunit was obtained only after $60 \mathrm{~min}$ in $100 \%$ $\mathrm{D}_{2} \mathrm{O}$. In order to observe a partial exchange situation for the $\varepsilon$ subunit, we have chosen an incubation time of 10 min.

The MALDI-MS spectra displayed in Figure 3 show the incorporation of deuterons upon incubation in various $\mathrm{D}_{2} \mathrm{O}$ /acetonitrile mixtures, as determined from the average mass measurement of the $\varepsilon$ subunit. Thus, 57 deuterons were incorporated after incubation in $100 \%$ $\mathrm{D}_{2} \mathrm{O}$ (Figure 3a and $\mathrm{b}$ ). When increasing amounts of acetonitrile were mixed with $\mathrm{D}_{2} \mathrm{O}$, the number of exchanged amide protons diminished: 52 deuterons were incorporated after an incubation in $\mathrm{D}_{2} \mathrm{O}$ with $20 \%$ of acetonitrile, 47 deuterons after incubation in $\mathrm{D}_{2} \mathrm{O}$ with $30 \%$ acetonitrile and 44 deuterons after an incubation in $\mathrm{D}_{2} \mathrm{O}$ with $40 \%$ acetonitrile (Figure $3 \mathrm{c}$ to e). In parallel to these experiments, a sample of $\varepsilon$ subunit obtained by solid phase synthesis [21] was analyzed by circular dichroism (CD) with an increasing concentration of acetonitrile. This study (data not shown) indicated that $\varepsilon$ subunit is mostly non-structured in neat water, and that an increase of acetonitrile concentration from 0 to $40 \%$ results in an increased $\alpha$-helical structuration for this polypeptide. Indeed, a $\Delta \varepsilon_{220 \mathrm{~nm}} / \Delta \varepsilon_{208 \mathrm{~nm}}$ ratio of about 0.8 , indicative of a single stranded helical structure, was found at $40 \%$ acetonitrile, whereas a possible peptide oligomerization would have given a value of
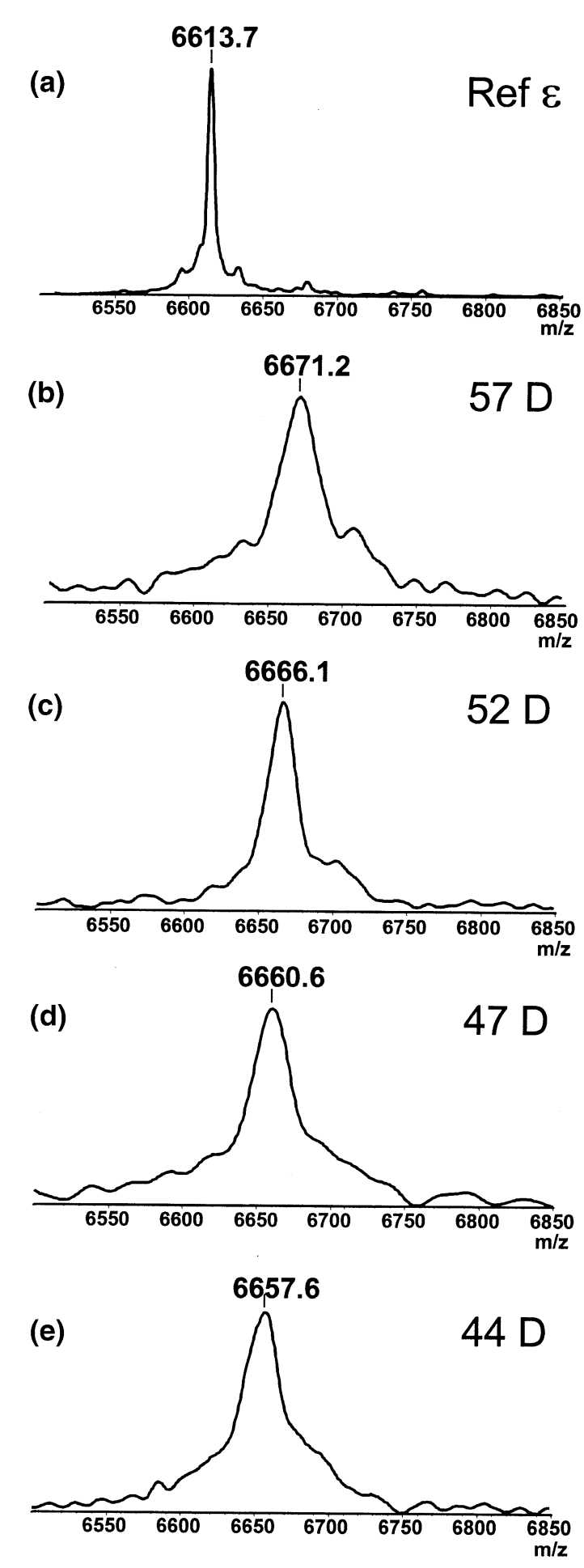

Figure 3. MALDI mass spectra of whole $\varepsilon$ subunit incubated in solutions containing $\mathrm{D}_{2} \mathrm{O}$ and different concentrations of acetonitrile. Isolated $\varepsilon$ subunit samples were prepared as described under Experimental. 25 picomoles of $\varepsilon$ subunit were incubated $10 \mathrm{~min}$ in the $\mathrm{D}_{2} \mathrm{O}$ solution at room temperature. After incubation, the exchange reaction was quenched $\left(\mathrm{pH} 2\right.$ and $\left.0{ }^{\circ} \mathrm{C}\right)$, the sample was mixed with matrix and analyzed by MALDI-MS in the linear mode. (a) Mass spectrum of reference $\varepsilon$ subunit (no incubation in $\mathrm{D}_{2} \mathrm{O}$ solution); (b) mass spectrum of $\varepsilon$ subunit after incubation in $100 \% \mathrm{D}_{2} \mathrm{O}$; (c), (d), (e) mass spectra of $\varepsilon$ subunit after incubation in $\mathrm{D}_{2} \mathrm{O}$ solution containing 20,30 , and $40 \%$ acetonitrile, respectively. 
about 1.0 [22]. Thus, the partial protection of exchangeable $\mathrm{H}$ atoms on $\varepsilon$ subunit observed with increasing acetonitrile concentration likely results from $\alpha$-helical structure, characterized by hydrogen bonding between amino acid residues involved in the helix. Noteworthy, acetonitrile concentrations above $40 \%$ led to a loss of helical structuration of $\varepsilon$ subunit, resulting in an increase of the H/D exchange (56 exchanged deuterons found at $70 \%$ acetonitrile concentration, close to the number found for the incubation in neat deuterium oxide.)

\section{Peptide Mapping of $\varepsilon$ Subunit}

In order to determine which regions of $\varepsilon$ subunit are most accessible to solvent, a peptide mass fingerprint was generated. The $\varepsilon$ subunit was cleaved using immobilized pepsin and the resulting peptides were analyzed by MALDI-MS [23]. The complex mixture of peptides is easily analyzed by MALDI-MS because $[\mathrm{M}+\mathrm{H}]^{+}$ions are by far the major ions observed for peptides in the 700-3000 Da mass range. The MALDI mass spectrum of the whole $\varepsilon$ subunit pepsin digest showed approximately 30 major peaks (Figure 4a). The major concern of spectrum processing was to assign an experimental mass to a given fragment without any ambiguity, while obtaining a maximal sequence coverage. All operations pertaining to this peptide mapping were performed with non-deuterated samples.

By means of MALDI-MS analysis alone, six peaks representing $89 \%$ of the primary sequence of $\varepsilon$ subunit could be assigned (Table 1). These selected peaks matched only one possible fragment belonging to $\varepsilon$ subunit, within a 20 ppm interval corresponding to the confidence limit of our external calibration system based on a mixture of eight reference peptides (see Experimental). All peaks that did contain a mixture of two or more peptides were not taken into consideration. Only the [3-20] segment could not be assigned with certainty by MALDI-MS alone. In this particular case, we could not apply PSD fragment analysis to obtain a related sequence tag because the resolving power of the ion gate of our MALDI-TOF instrument did not allow a proper isolation of the peptides that could account for this peptide stretch. Therefore, we have used combined LC/MS/MS on an ion trap to identify the $\mathrm{m} / \mathrm{z} 1953.06$ peak, which turned out to correspond to an unique peptide in the digest, thus completing the sequence coverage of the $\varepsilon$ subunit.

Assigning and selecting the peptide peaks that allow counting deuteron incorporation along a polypeptide sequence represents the most time consuming part of HXMS experiments relying on MALDI-MS. Extending this kind of study to larger subunits belonging to a supramolecular complex often requires the assistance of LC/MS/MS for unambiguous peptide mapping, an interesting alternative certainly being the use of MALDI-TOF/TOF instruments with improved ion gates.

\section{Solvent Accessibility in F1-ATPase Complex}

Once the peptide mapping was completed, the H/D exchanged $\varepsilon$ subunit was submitted to proteolytic cleavage with pepsin. Subsequently, the deuteration level of each selected peptide fragment was determined from the mass spectrum of the complex mixture (Figure $4 b)$. For this, the centroids of the mass envelope for the undeuterated and deuterated peptide were calculated $[24,25]$. As a control, the $\varepsilon$ subunit was isolated from F1-ATPase by means of fast micro-chromatography and cleaved by immobilized pepsin. The resulting fragments were dried and incubated in $100 \% \mathrm{D}_{2} \mathrm{O}$ for 30 min to allow exchange control and the amount of deuterium incorporated by each assigned peak was determined. For each peptide, 80 to $90 \%$ of the amide protons were exchanged by deuterium after $30 \mathrm{~min}$. This value corresponds to the maximal experimental exchange level observed for $\varepsilon$ subunit peptides after 30 min, because neither secondary structuration nor supramolecular interactions may diminish the exchange level (Figure 5b).

To confirm the hypothesis of the influence of $\varepsilon$ subunit $\alpha$-helical structuration on deuterium labeling, the $\varepsilon$ subunit previously isolated by chromatography was incubated $10 \mathrm{~min}$ in $\mathrm{D}_{2} \mathrm{O}$ in presence of $40 \%$ acetonitrile. After this time, the $\varepsilon$ subunit was digested and the incorporation of deuterium was determined for each peptide. The [3-20] peptide displayed the lowest deuterium incorporation (Figure 5a). These data are in full agreement with the existence of an $\alpha$-helix for the [13-23] region in the 3-D structure of the bovine $\varepsilon$ subunit of ATPase [17].

In a final experiment, native F1-ATPase was submitted to HXMS in order to determine which regions of $\varepsilon$ subunit are the most accessible to deuterium oxide. Thus, these experiments used a buffered medium for $H / D$ exchange in order to maintain a high enzymatic activity level for the F1-ATPase complex (cf Experimental for assay conditions). A kinetic study indicated that after $20 \mathrm{~min}$ incubation time, the deuterium content remained constant for the $\varepsilon$ subunit. This strongly underlines the importance of supramolecular interactions for the incorporation of deuterium. The highest exchange level was found for the [24-40] peptide, while the less exchangeable peptide was the [3-20] (Figure $5 b)$.

A 3-D structure of yeast F1-ATPase not being available, we evaluated the results of our H/D exchange experiments by means of the known structure of the bovine enzyme. Indeed, the sequence alignment of the yeast $\varepsilon$ subunit with its bovine counterpart is straightforward: a large homology region comprising the highly structured core of the bovine subunit encompasses the three $\alpha$-helices (59\% homology with $44 \%$ strict identity; see Figure 6).

Since there was no data available on the structure of the C-terminal part of the yeast $\varepsilon$ subunit, we used secondary prediction softwares (cf Experimental sec- 

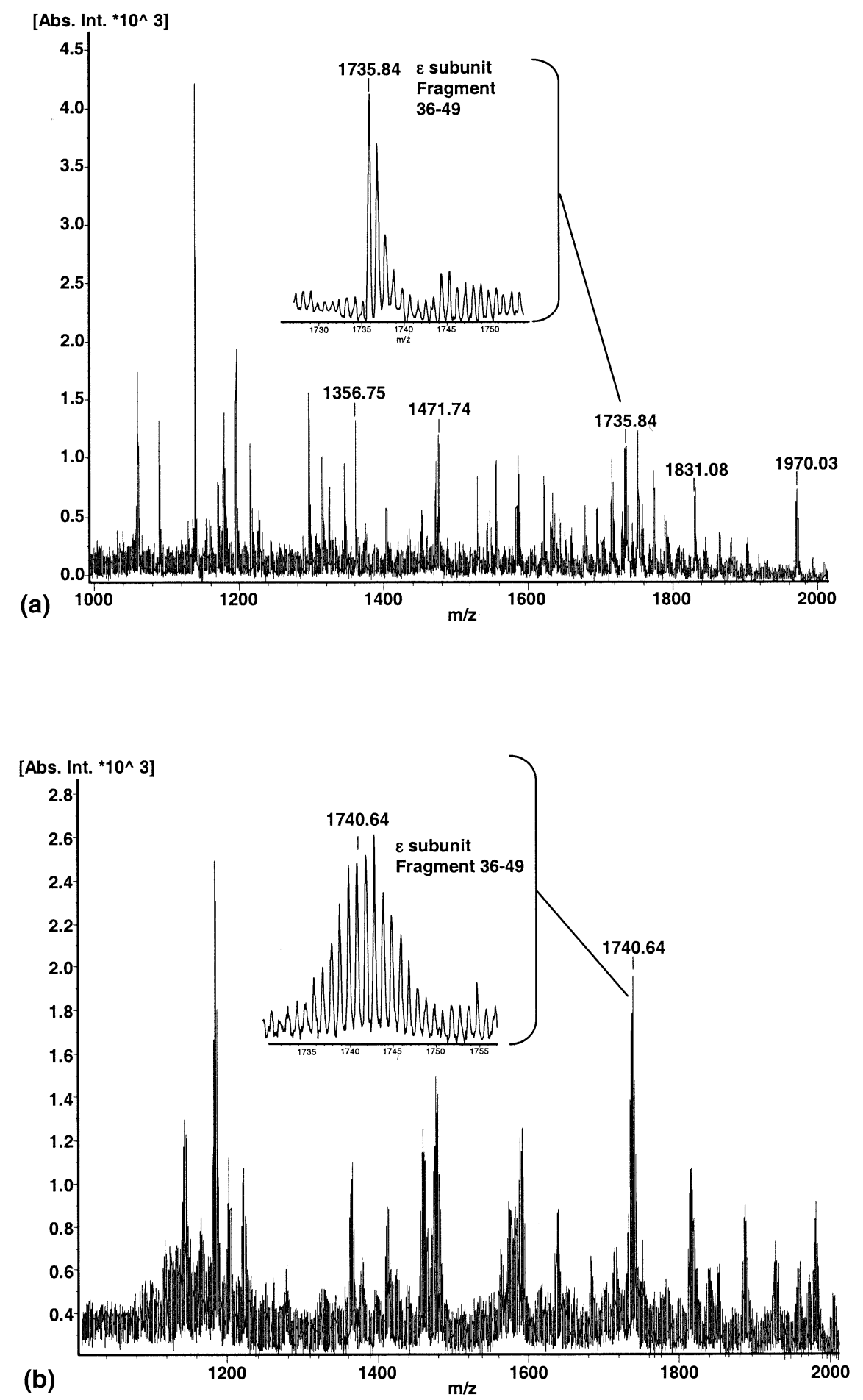

Figure 4. (a) MALDI mass spectrum of the peptide mixture obtained after pepsin digestion of non-deuterated $\varepsilon$ subunit. The inset is an expansion of the peptide peak 1735.84 (fragment [36-49]). (b) MALDI mass spectrum of the mixture obtained after pepsin digestion of deuterated $\varepsilon$ subunit following a 30 min incubation of 30 pmol ATPase in $\mathrm{D}_{2} \mathrm{O}$. After fast micro-chromatography, the isolated $\varepsilon$ subunit was cleaved using immobilized pepsin and analyzed in the same conditions as in (a). The inset is an expansion of the peptide peak corresponding to the deuterated fragment [36-49]. Mass values correspond to the centroid of the envelope of the mass peaks for $[\mathrm{M}+\mathrm{H}]^{+}$ pseudo-molecular ions.

tion). Identical results were obtained in all cases, with an accurate prediction of the three $\alpha$-helices of the $\mathrm{N}$-terminal part: On average from A2 to A6, from S9 to
$\mathrm{L} 25$, and from Q30 to L35, corresponding to the X-ray structure (h1 from S1 to A6, h2 from S9 to S24, and h3 from E28 to V34). Conversely, none of these software- 
Table 1. Assignment of peptide ions covering the sequence of $\varepsilon$ subunit

\begin{tabular}{|c|c|c|c|c|}
\hline $\begin{array}{l}\text { Calculated } \\
\text { mass }^{\mathrm{a}}\end{array}$ & $\begin{array}{l}\text { Measured } \\
\text { mass }^{a}\end{array}$ & Position & Sequence & $\begin{array}{l}\text { Identification } \\
\text { method }^{\text {b }}\end{array}$ \\
\hline 1356.71 & 1356.72 & $2-13$ & AWRKAGISYAAY & Exact mass \\
\hline 1953.04 & 1953.04 & $3-20$ & WRKAGISYAAYLNVAAQA & LC/MS/MS \\
\hline 1831.03 & 1831.06 & $20-36$ & AIRSSLKTELQTASVLN & Exact mass \\
\hline 1876.01 & 1876.03 & $24-40$ & SLKTELQIASVLNRSQT & Exact mass \\
\hline 1471.72 & 1471.73 & $32-44$ & ASVLNRSQTDAFY & Exact mass \\
\hline 1735.80 & 1735.82 & $36-49$ & NRSQTDAFYTQYKN & Exact mass \\
\hline 1969.99 & 1970.00 & $44-61$ & YTQYKNGTAASEPTPITK & Exact mass \\
\hline
\end{tabular}

${ }^{\mathrm{a}} \mathrm{MH}^{+}$monoisotopic masses.

${ }^{b}$ Exact mass indicates that only one possible peptide from $\varepsilon$ subunit had a mass that matched the experimental mass within 20 ppm.

aided predictions indicated a secondary structure for the C-terminal domain of the sequence, but only an
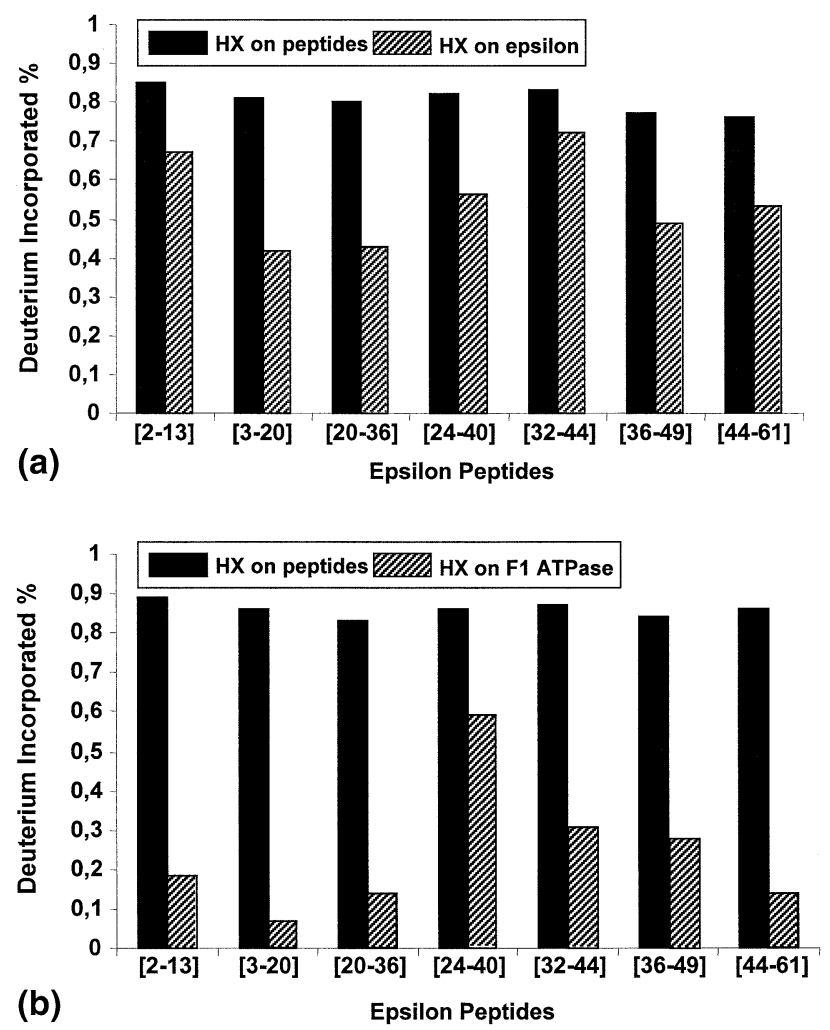

Figure 5. (a) Effect of $\alpha$-helical structure on H/D exchange level along the primary structure of the $\varepsilon$ subunit. The deuterium incorporated (\%) is calculated for each $\varepsilon$ fragment $\left(\right.$ as $\mathrm{D} / \mathrm{H}_{\mathrm{t}}$, where $D$ is the measured amount of deuterium incorporated and $\mathrm{H}_{t}$ is the total of exchangeable hydrogens for the fragment). These histograms result from four independent experiments (s \# 0.015). HXMS on peptides: A pepsin digest of $\varepsilon$ subunit was incubated 10 min in $\mathrm{D}_{2} \mathrm{O}$ :acetonitrile 6:4. HXMS on $\varepsilon$ : isolated $\varepsilon$ subunit was submitted to $\mathrm{H} / \mathrm{D}$ exchange (10 $\mathrm{min}$ in $\mathrm{D}_{2} \mathrm{O}$ :acetonitrile 6:4) and then digested with pepsin. (b) Effect of protein-protein interactions within the ATPase complex on H/D exchange level along the primary structure of the $\varepsilon$ subunit. The deuterium incorporated (\%) is calculated as in the case of (a). These histograms result from four independent experiments (s \# 0.015). HXMS on peptides: A pepsin digest of $\varepsilon$ subunit was incubated $30 \mathrm{~min}$ in $\mathrm{D}_{2} \mathrm{O}$, $0.04 \mathrm{M} \mathrm{MgSO}_{4}, 50 \mathrm{mM}$ Tris $\mathrm{SO}_{4}$. HX on F1 ATPase: The F1-ATPase complex was incubated $30 \mathrm{~min}$ in $\mathrm{D}_{2} \mathrm{O}, 0.04 \mathrm{M} \mathrm{MgSO}_{4}, 50 \mathrm{mM}$ Tris $\mathrm{SO}_{4}$, then the $\varepsilon$ subunit was isolated by fast micro-chromatography and submitted to pepsin digestion. extended conformation for the part between D41/A42 and $\mathrm{Y} 47 / \mathrm{K} 48$, the remaining part of the sequence predicted to be a random coil.

A sharp and highly lipophilic zone appears clearly around $\mathrm{W} 3, \mathrm{Y10}$, and $\mathrm{Y} 13$ residues, in a well structured part of the protein. As these three aromatic moieties are in close spatial proximity, it is very likely that this area is involved in protein-protein interaction.

In order to visualize which regions of $\varepsilon$ subunit are the most accessible to deuterium in F1-ATPase complex, a model based on the bovine structure reflecting the sequence homology and secondary structure prediction has been built: Thus, the structured [1-44] part is followed by an extended C-terminal domain (Figure 7a). The grey scale encoding shows the highest $\mathrm{H} / \mathrm{D}$ exchange level localized on the [24-27] turn and [2837] h3 $\alpha$-helix. Further, a model structure of the three neighboring subunits $\varepsilon, \gamma$, and $\delta$ has been built on the basis of the bovine structure, the yeast $\varepsilon$ subunit replacing its bovine homologous subunit (Figure $7 \mathrm{~b}$ ). This model shows the interaction domains between subunits, and is in excellent agreement with our observation of the highest H/D exchange level located in the [24-40] region.

\section{Conclusion}

Hydrogen/deuterium exchange monitored by MALDI mass spectrometry is a well-performing technique to study the topology of supramolecular hetero-oligomeric complexes. The use of fast reversed phase microchromatography solved the problem of deuterium back exchange that may result from the steps required for complex disruption and subunit purification. HXMS was used to study the kinetics of deuterium incorporation in ATPase $\varepsilon$ subunit, the effect of secondary structure on deuterium incorporation and finally the accessibility of $\varepsilon$ to solvent within the native complex. The results establish the ability of MALDI-HXMS to provide useful information on the conformational state of a protein and on its supramolecular interactions, while only requiring a few picomoles of sample. This approach may be extended to larger subunits and other supramolecular complexes, provided that target sub- 


\section{E_YEAST : SAWRKAGISYAAYLNVAAQAIRSSLKTELQTASYLNRSQTDAFYTQYKNGTAASEPTPITK EPS_BOV :VAYWRQAGLSYIRYSQICAKAVRDALKTEFKANAMKTSGSTIKIVKV}

Figure 6. Sequence alignment of bovine and yeast $\varepsilon$ subunits; the frame shows the highest homology area.

(a)
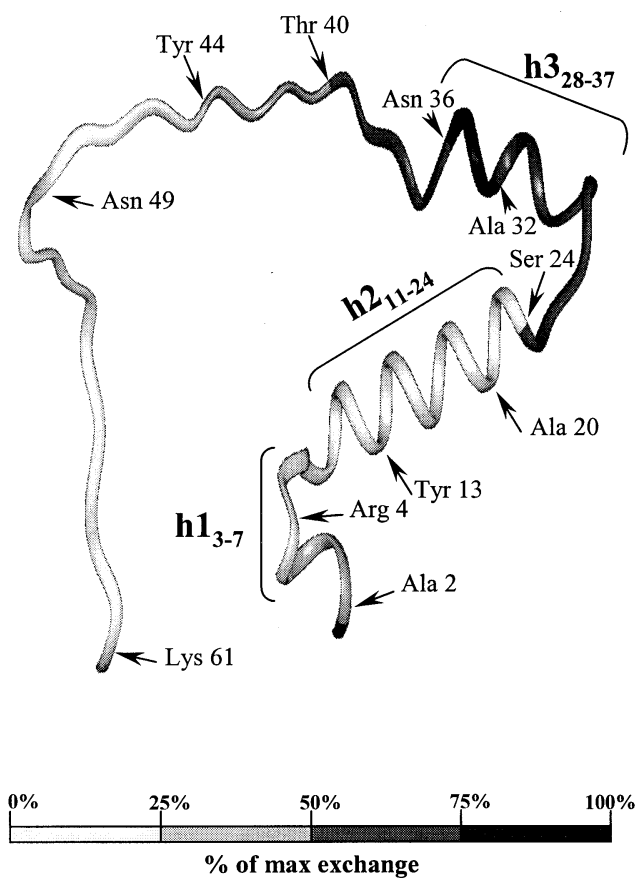

(b)

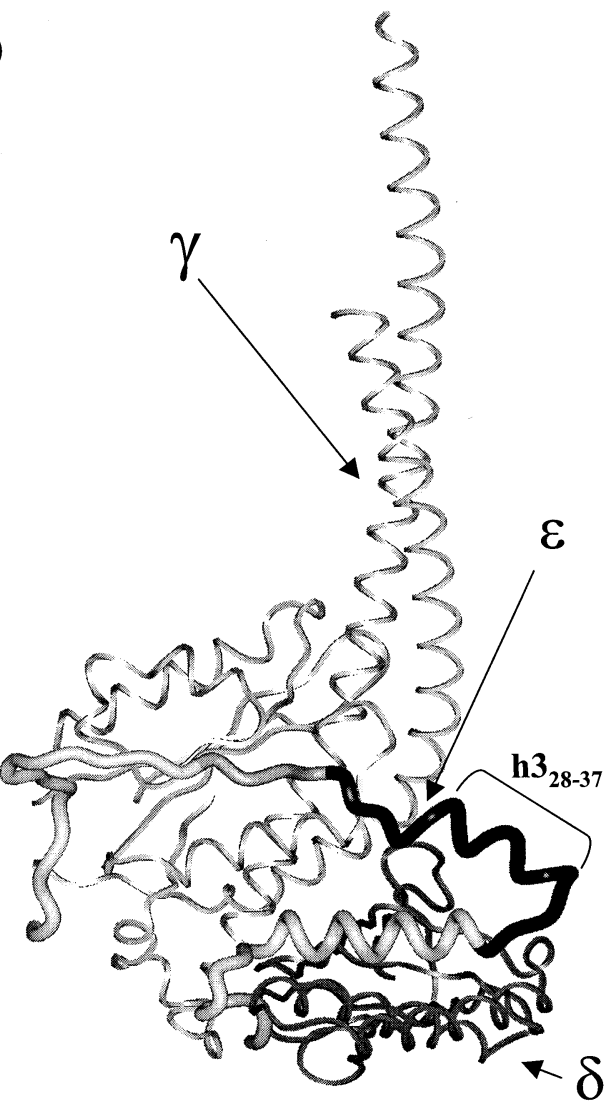

Figure 7. Representation of $\varepsilon$ subunit accessibility to $\mathrm{D}_{2} \mathrm{O}$ in F1-ATPase. (a) A model structure of the yeast $\varepsilon$ subunit has been built on the basis of the bovine enzyme for the first 47 aminoacids, leaving a random coil for the remaining amino acids that do not exist in the bovine enzyme. Four levels of $\mathrm{H} / \mathrm{D}$ exchange are indicated from very low level in white to high level in black. (b) The yeast $\varepsilon$ subunit model structure (instead of its corresponding bovine subunit) is shown in interaction with the neighboring $\gamma$ and $\delta$ subunits. This scheme reveals a [24-37] domain highly exposed to the solvent, in agreement with the highest measured H/D exchange levels.

units can be separated by fast chromatography, and that peptide mapping is conducted carefully in order to deliver unambiguous sequence coverage. MALDI-MS alone might not be accurate enough to achieve successfully this task in all cases, and should then be associated to MS/MS sequence assignment for this first part of HXMS methodology.

\section{Acknowledgments}

The authors gratefully acknowledge the financial support of CNRS (ACI program "Dynamique et réactivité des assemblages biologiques") and of the Région Aquitaine, and the help of Dr. Marc Bonneu for ion trap mass measurements.

\section{References}

1. Woodward, C. K. Hydrogen Exchange Rates and Protein Folding. Curr. Opin. Struct. Biol. 1994, 4, 112-116.

2. Hvidt, A. Hydrogen Exchange in Proteins. Adv. Protein Chem. 1966, 21, 287-385.

3. Miranker, A.; Robinson, C. V.; Radford, S. E.; Aplin, R. T.; Dobson, C. M. Detection of Transient Protein Folding Populations by Mass Spectrometry. Science 1993, 262, 896-900.

4. Englander, S.; Mayne, W.; Bai, Y.; Sosnick, T. R. Hydrogen Exchange: The Modern Legacy of Linderstrom-Lang. Protein Sci. 1997, 6, 1101-1109.

5. Hoofnagle, A.; Resing, K.; Goldsmith, E.; Nathalie, G. Changes in Protein Conformational Mobility Upon Activation of Extracellular Regulated Protein Kinase-2 as Detected by Hydrogen Exchange. Proc. Natl. Acad. Sci. U.S.A. 2000, 98, 956-961. 
6. Englander, J. J.; Rogero, J. R.; Englander, S. W. Protein Hydrogen Exchange Studied by the Fragment Separation Method. Anal. Biochem. 1985, 147, 234-244.

7. Karas, M.; Bachmann, D.; Bahr, U.; Hillenkamp, F. MatrixAssisted Ultraviolet Laser Desorption of Non-Volatile Compounds. Int. J. Mass Spectrom. Ion Processes 1987, 78, 53-68.

8. Burlingame, A. L.; Boyd, R. K.; Gaskell, S. J. Mass Spectrometry. Anal. Chem. 1994, 68, 634R-683R.

9. Mandell, J. G.; Falick, A. M.; Komives, E. A. Measurement of Amide Hydrogen Exchange by MALDI-TOF Mass Spectrometry. Anal. Chem. 1998, 70, 3987-3995.

10. Figuerosa, I.; Russel, D. Matrix-Assisted Laser Desorption/ Ionization Hydrogen/Deuterium Exchange Studies to Probe Peptide Conformational Changes. J. Am. Soc. Mass. Spectrom. 1999, 10, 719-731.

11. Arselin, G.; Gandar, J. C.; Guérin, B.; Velours, J. Isolation and Complete Amino Acid Sequence of the Mitochondrial ATP Synthase $\varepsilon$-Subunit of the Yeast Saccharomyces cerevisiae. J. Biol. Chem. 1991, 266, 723-727.

12. Daum, G.; Böhni, P. C.; Schatz, G. Import of Proteins into Mitochondria. Cytochrome b2 and Cytochrome c Peroxidase are Located in the Intermembrane Space of Yeast Mitochondria. J. Biol. Chem. 1982, 257, 13023-13033.

13. Beechey, R. B.; Hubbard, S. A.; Linnet, P. E.; Mitchell, A. D.; Munn, E. A Simple and Rapid Method for the Preparation of Adenosine Triphosphatase from Submitochondrial Particles. Biochem. J. 1975, 148, 533-537.

14. Penin, F.; Godinot, C.; Gautheron, D. C. Optimization of the Purification of Mitochondrial $\mathrm{F}_{1}$-Adenosine Triphosphatase. Biochim. Biophys. Acta. 1979, 548, 63-71.

15. Falson, P.; Di Pietro, A.; Gautheron, D. C. Chemical Modification of Thiol Groups of Mitochondrial F1-ATPase from the Yeast Schizosaccharomyces pombe. J. Biol. Chem. 1986, 261, 71517159.
16. Penefsky, H. S. Reversible Binding of Pi by Beef Heart Mitochondrial Adenosine Triphosphatase. J. Biol. Chem. 1977, 252, 2891-2899.

17. Gibbons, C.; Montgomery, M. G.; Lesli, A. G. W.; Walker, J. E. The Structure of the Central Stalk in Bovine F1-ATPase at 2.4 Å Resolution. Nat. Struct. Biol. 2000, 7, 1055-1061.

18. Laguerre, M.; Saux, M.; Dubost, J. P.; Carpy, A. MLPP: A Program for Calculation of Molecular Lipophilicity in Proteins. Pharmacol. Sciences 1997, 3, 217-222.

19. Liu, Y. Q.; Smith, D. L. Probing High Order Structure of Proteins by Fast-Atom Bombardment Mass Spectrometry. J. Am. Soc. Mass. Spectrom. 1994, 5, 19-28.

20. Demmers, J.; Haverkamp, J.; Heck, A.; Koeppe, R.; Killian, A. Electrospray Ionization Mass Spectrometry as a Tool to Analyze Hydrogen/Deuterium Exchange Kinetics of Transmembrane Peptides in Lipid Bilayers. Proc. Natl. Acad. Sci. U.S.A. 2000, 97, 3189-3194.

21. Aznar-Derunes, C.; Manigand, C.; Picard, P.; Dautant, A.; Goetz, M.; Schmitter, J. M.; Precigoux, G. Study of the Yeast Saccharomyces cerevisiae F1F0-ATPase $\varepsilon$-Subunit. J. Pep. Sci. 2002, 8, 365-372.

22. Goetz, M.; Carlotti, C.; Bontems, F.; Dufourc, E. J. Evidence for an $\alpha$-helix- $>\pi$-Bulge Helicity Modulation for the neu/erbB-2 Membrane-Spanning Segment. A ${ }^{1} \mathrm{H}$ NMR and Circular Dichroism Study. Biochemistry 2001, 40, 6534-6540.

23. Juhasz, P.; Martin, S. A. The Utility of Nonspecific Proteases for Characterization of Glycoproteins by High-Resolution Time-of-Flight Mass Spectrometry. Int. J. Mass. Spectrom. Ion Processes 1997, 169, 217-230.

24. Mardsen, J. E.; Tromba, A. J. Vector Calculus; 3rd ed. W. H. Freeman and Co: New York, 1988, Chapter VI.

25. Zhang, Z.; Smith, D. Determination of Amide Hydrogen Exchange by Mass Spectrometry: A New Tool for Protein Structure Elucidation. Protein Sci. 1993, 2, 522-531. 